\title{
Quasibound states in type-II ZnTe/CdSe superlattices studied by modulation spectroscopies and photoconductivity at room temperature
}

\author{
S. M. Tseng and Y. F. Chen \\ Department of Physics, National Taiwan University, Taipei, Taiwan, Republic of China \\ Y. T. Cheng, C. W. Hsu, and Y. S. Huang \\ Department of Electronic Engineering, National Taiwan University of Science and Technology, Taipei, Taiwan, Republic of China \\ D. Y. Lin \\ Department of Electronic Engineering, Nan Kai College of Technology and Commerce, Tsao-Tun, Taiwan, Republic of China
}

(Received 12 February 2001; published 15 October 2001)

\begin{abstract}
We report that the quasibound states at the above-barrier region in type-II ZnTe/CdSe superlattices can be clearly observed at room temperature by photoreflectance, contactless electroreflectance, as well as photoconductivity measurements. We provide concrete evidence to confirm that free-carrier confinement at barrier layer (either in the valence-band CdSe layer or in the conduction-band ZnTe layer) does exist. It is found that the barrier-width dependence of the above-barrier ground-state transition energies can be described well by the constructive interference condition. We also observe the absorptive spatially indirect transition between electrons confined in the CdSe and holes confined in the ZnTe layers.
\end{abstract}

DOI: 10.1103/PhysRevB.64.195311

PACS number(s): 78.66.Hf

\section{INTRODUCTION}

The confinement of electrons and holes in semiconductor quantum wells and superlattices has been investigated using a variety of experimental techniques and theoretical methods. It has stimulated many important phenomena as well as industrial applications. In optical studies carried out so far, attention has been focused primarily on excitons involving electron and hole states at energies below the barriers, which are necessarily localized in their respective quantum well regions. Recently, the existence of above-barrier quasibound states has been observed by different experimental methods, such as Raman spectroscopy, ${ }^{1}$ photoluminescence excitation spectroscopy, ${ }^{2}$ absorption spectroscopy, ${ }^{2-4}$ photoreflectance (PR) spectroscopy, ${ }^{5,6}$ and piezoreflectance spectroscopy. ${ }^{7}$ Most studies related to above-barrier quasibound states have been focused on type-I superlattices. ${ }^{3}$ It is expected that quasilocalized states above potential barriers can also have a significant effect on optical properties of type-II semiconductor heterostructures. There are several studies devoted to this behavior, however, most previous studies were performed at very low temperature, and a clear verification of the barrierwidth dependence of the transition energies has not been reported, to the best of our knowledge. ${ }^{4,8,9}$ In this paper, we present clear experimental evidence for the existence of above-barrier quasibound states in type-II ZnTe/CdSe superlattices at room temperature using $\mathrm{PR}$, contactless electroreflectance (CER), as well as photoconductivity (PC) measurements. We show that the barrier-width dependence of abovebarrier ground states can be well described by the constructive interference model. We also report the observation of the absorptive spatially indirect transition between electrons confined in the CdSe and holes confined in the ZnTe layers.

\section{EXPERIMENT}

The multiple quantum well structures were grown by molecular-beam epitaxy on (100) GaAs substrates after the deposition of about 1.26-2 $\mu \mathrm{m}$ of a ZnTe buffer layer. Details of the growth were described elsewhere. ${ }^{10,11}$ The relevant sample parameters are summarized in Tables I and II. All superlattices used here contain 20 periods of alternating ZnTe and CdSe layers.

The characters of above-barrier quasibound states in ZnTe/CdSe superlattices were determined by PR, CER, and PC measurements. The experimental details of the PR measurements can be found elsewhere. ${ }^{12}$ In our PR measurement, a 30-mW Nd: YAG (yttrium aluminum garnet) laser was used as the modulating source chopped at $200 \mathrm{~Hz}$. The laser intensity was reduced to about $10 \%$ of its initial value by using a neutral density filter. A $150-\mathrm{W}$ tungsten-halogen

TABLE I. Transition energies (eV) for the CdSe layer. The transitions are labeled with the first number indicating the conductionband state and the second the valence-band state, with " $H$ " and " $L$ " indentifying heavy and light holes. The above-barrier transitions, which involve quasiconfined states, are denoted by a subscript " $q$ " after the number. Note that the theoretical values are corrected for excitonic binding energies.

\begin{tabular}{|c|c|c|c|c|c|}
\hline $\begin{array}{l}\text { Sample } \\
\text { name }\end{array}$ & $\begin{array}{l}\text { ZnTe layer } \\
\text { width (nm) }\end{array}$ & $\begin{array}{l}\text { CdSe layer } \\
\text { width (nm) }\end{array}$ & $\begin{array}{c}\text { Intersubb } \\
\text { Transition }\end{array}$ & $\begin{array}{l}\text { and characte } \\
\text { Experiment } \\
(\mathrm{PR})(\mathrm{eV})\end{array}$ & $\begin{array}{l}\text { istics } \\
\text { Theory } \\
(\mathrm{eV})\end{array}$ \\
\hline \multirow[t]{2}{*}{ A } & 15 & 21 & $11_{q} H$ & 1.678 & 1.677 \\
\hline & & & $11_{q} L$ & 1.682 & 1.681 \\
\hline \multirow[t]{2}{*}{ B } & 15 & 9 & $11_{q} H$ & 1.708 & 1.698 \\
\hline & & & $11_{q} L$ & 1.726 & 1.719 \\
\hline \multirow[t]{2}{*}{$\mathrm{C}$} & 15 & 6.9 & $11_{q} H$ & 1.732 & 1.729 \\
\hline & & & $11_{q} L$ & 1.769 & 1.764 \\
\hline \multirow[t]{2}{*}{ D } & 9 & 9 & $11_{q} H$ & 1.693 & 1.698 \\
\hline & & & $11_{q} L$ & 1.719 & 1.719 \\
\hline \multirow[t]{2}{*}{$\mathrm{E}$} & 24 & 9 & $11_{q} H$ & 1.699 & 1.698 \\
\hline & & & $11_{q} L$ & 1.721 & 1.719 \\
\hline
\end{tabular}


TABLE II. Transition energies $(\mathrm{eV})$ for the $\mathrm{ZnTe}$ layer. The transitions are labeled with the first number indicating the conduction-band state and the second the valence-band state, with " $H$ " and " $L$ " indentifying heavy and light holes. The above-barrier transitions, which involve quasiconfined states, are denoted by a subscript " $q$ " after the number. Note that the theoretical values are corrected for excitonic binding energies.

\begin{tabular}{|c|c|c|c|c|c|}
\hline \multirow[b]{2}{*}{$\begin{array}{l}\text { Sample } \\
\text { name }\end{array}$} & \multirow[b]{2}{*}{$\begin{array}{l}\text { ZnTe layer } \\
\text { width (nm) }\end{array}$} & \multirow[b]{2}{*}{$\begin{array}{l}\text { CdSe layer } \\
\text { width (nm) }\end{array}$} & \multicolumn{3}{|c|}{ Intersubband characteristics } \\
\hline & & & Transition & $\begin{array}{l}\text { Experiment } \\
(\mathrm{CER})(\mathrm{eV})\end{array}$ & $\begin{array}{l}\text { Theory } \\
(\mathrm{eV})\end{array}$ \\
\hline \multirow[t]{2}{*}{ A } & 15 & 21 & $1_{q} 1 H$ & 2.261 & 2.254 \\
\hline & & & $1_{q} 1 L$ & 2.278 & 2.263 \\
\hline \multirow[t]{2}{*}{$\mathrm{E}$} & 24 & 9 & $1_{q} 1 H$ & 2.254 & 2.244 \\
\hline & & & $2{ }_{q} 2 H$ & 2.271 & 2.262 \\
\hline \multirow[t]{2}{*}{$\mathrm{F}$} & 15 & 15 & $1_{q} 1 H$ & 2.262 & 2.254 \\
\hline & & & $1_{q} 1 L$ & 2.277 & 2.261 \\
\hline
\end{tabular}

lamp filtered by a model 270 McPherson 0.35-m monochromator provided the monochromatic light. The reflected light was detected by an EG\&G type HUV-2000B silicon photodiode, and the signal was recorded from an NF model 5610B lock-in amplifier.

The details of the CER method, which utilized a condenserlike system, have already been reported. ${ }^{13}$ Here an ac modulating voltage (500-1000 V peak-to-peak) at a frequency of $200 \mathrm{~Hz}$ was employed. A $150 \mathrm{~W}$ xenon arc lamp filtered by a PTI $0.25-\mathrm{m}$ monochromator provided the monochromatic light. The reflected light was detected by an EMR model 541E photomultiplier tube (PMT). The dc output of the PMT was maintained constant throughout the experiment by an electronic sevo, which controls the high voltage applied to the PMT. The signal was recorded by a Stanford Research model SR830 lock-in amplifier.

For the PC measurement, Ohmic contacts were formed by depositing indium drops at the four corners of the samples, and annealing the samples at $240^{\circ} \mathrm{C}$ for $10 \mathrm{~min}$. A tungsten lamp dispersed by an ARC Spectra Pro-275 triple-grating monochromator was used as the photoexcitation light source. The photoexcitation light was chopped by a mechanical chopper at a frequency of $200 \mathrm{~Hz}$. The light beam was focused on the sample by a $10-\mathrm{cm}$ focal length lens. A constant current was supplied to the sample by a Keithley 236 source measure unit. The conductivity signal was detected as a change in the voltage drop across the sample using a lock-in amplifier.

\section{THEORETICAL BACKGROUND}

The line shapes of the modulation spectroscopies can be expressed as ${ }^{13,14}$

$$
\frac{\Delta R}{R}=\alpha\left(\varepsilon_{1}, \varepsilon_{2}\right) \Delta \varepsilon_{1}+\beta\left(\varepsilon_{1}, \varepsilon_{2}\right) \Delta \varepsilon_{2}
$$

where $\alpha$ and $\beta$ are Seraphin coefficients and $\Delta \varepsilon_{1}$ and $\Delta \varepsilon_{2}$ are, respectively, the photomodulated real and imaginary

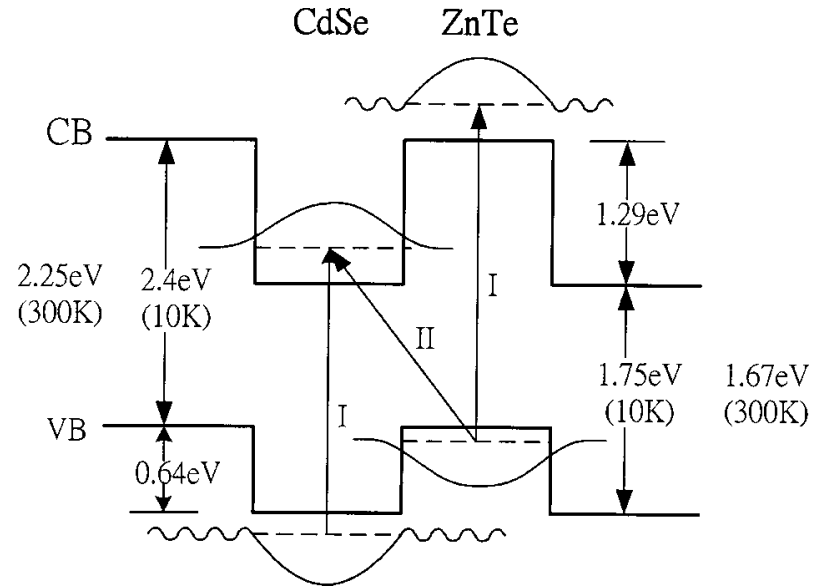

FIG. 1. Schematic diagram of the band alignment for $\mathrm{ZnTe} /$ CdSe superlattices. Dashed lines indicate confined electron (hole) states in the CdSe (ZnTe) layers, as well as the above-barrier localized electron (hole) in the $\mathrm{ZnTe}(\mathrm{CdSe})$ layers. The wave functions of the conduction- (valence-) band ground state and the lowest above-barrier hole (electron) state are plotted. The arrows indicate the spatially direct and indirect transitions in this type-II system.

components of the complex dielectric function $\varepsilon=\varepsilon_{1}+i \varepsilon_{2}$. The terms $\Delta \varepsilon_{1}$ and $\Delta \varepsilon_{2}$ are related by a Kramers-Kronig relation.

It has been shown that for bound states such as excitons or the confined states of a quantum well, $\Delta \varepsilon$ is a firstderivative spectroscopy. For excitons, the dielectric function will have either a Lorentzian or Gaussian profile, depending on whether the broadening is homogeneous or inhomogeneous, respectively. The details of the line-shape considerations can be found in Refs. 13, 15, and 16.

The energy-band alignment for $\mathrm{ZnTe} / \mathrm{CdSe}$ superlattices is schematically shown in Fig. 1. ${ }^{8,17}$ The valence-band offset for the $\mathrm{ZnTe} / \mathrm{CdSe}$ superlattices is $\Delta E=0.64 \pm 0.07 \mathrm{eV}$ at room temperature, ${ }^{18}$ as determined by $\mathrm{x}$-ray photoelectron spectroscopy. Two types of excitonic transitions are possible in such type-II superlattices. One takes place between electron states localized in the CdSe conduction-band wells and hole states in the ZnTe wells, as shown by the arrow marked "II" in Fig. 1. We shall refer to this process, involving spatially separated states, as a type-II excitonic transition. The other occurs between electron (or hole) subbands confined in the wells and hole (or electron) subbands at above-barrier energies, quasiconfined in the barriers. In that case both the electron and the hole states involved in the transition are localized in the same layer, as shown by the arrows marked "I" in Fig. 1. We shall refer to this spatially direct process as a type-I excitonic transition. ${ }^{17}$ For example, in the valence band, the CdSe layer acts as a barrier, in which states can be quasibound. The portions of the wave functions of the lowest-energy quasibound states in the CdSe valence-band barrier are similar to those of the corresponding truly localized states in the conduction-band well. The similarity of the wave functions leads to large wave-function overlap and, thus, strong optical transitions occur. ${ }^{19}$ In this study, we will concentrate our attention on the transitions in the barrier region. 


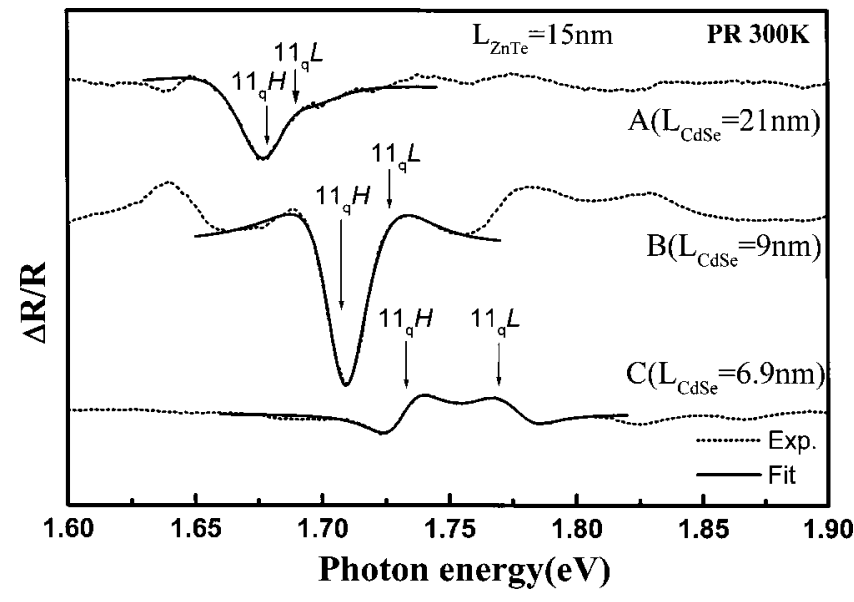

FIG. 2. The PR spectra of samples A, B, and C at $300 \mathrm{~K}$. The dotted lines represent experimental results, and solid lines are obtained by least-squares fits of a line-shape function.

\section{RESULTS AND DISCUSSION}

Figure 2 shows the PR spectra at room temperature for samples A, B, and C with CdSe layer widths (labeled " $L_{\mathrm{CdSe}}$ ") of 21,9 , and $6.9 \mathrm{~nm}$, respectively, and the $\mathrm{ZnTe}$ layer width fixed to $15 \mathrm{~nm}$ (labeled " $L_{\mathrm{ZnTe}}$ "). Figure 3 shows the PR spectra at room temperature for samples D and E with the CdSe layer width fixed to $9 \mathrm{~nm}$, and ZnTe layer widths of 9 and $24 \mathrm{~nm}$, respectively. The transitions indicated by arrows are obtained from the least-squares fits of a line-shape function to the experimental data. The dotted and solid lines represent the experimental and fitted results, respectively. Details of the line shape were discussed in Ref. 6. For labeling the transitions, we use the notation in which the first number indicates the conduction-band state and the second the valence-band state, with " $H$ " and " $L$ " identifying heavy and light holes. The above-barrier transitions that involve quasibound states are denoted by a subscript $q$ after the number. A detailed comparison between the transition energies of experimental and theoretical results will be given below. We can clearly observe that the main feature of the PR spectra is

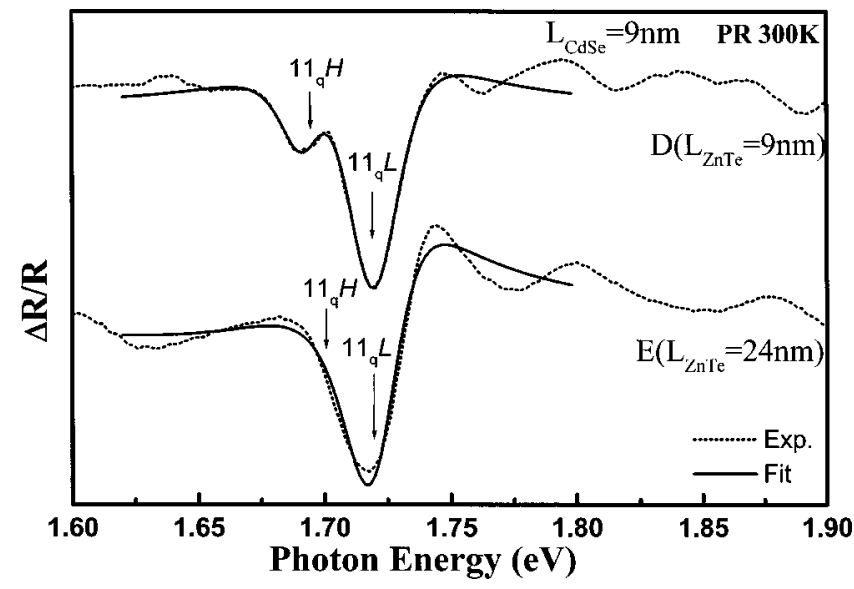

FIG. 3. The PR spectra of samples D and E at $300 \mathrm{~K}$. The dotted lines represent experimental results, and solid lines are obtained by least-squares fits of a line-shape function.

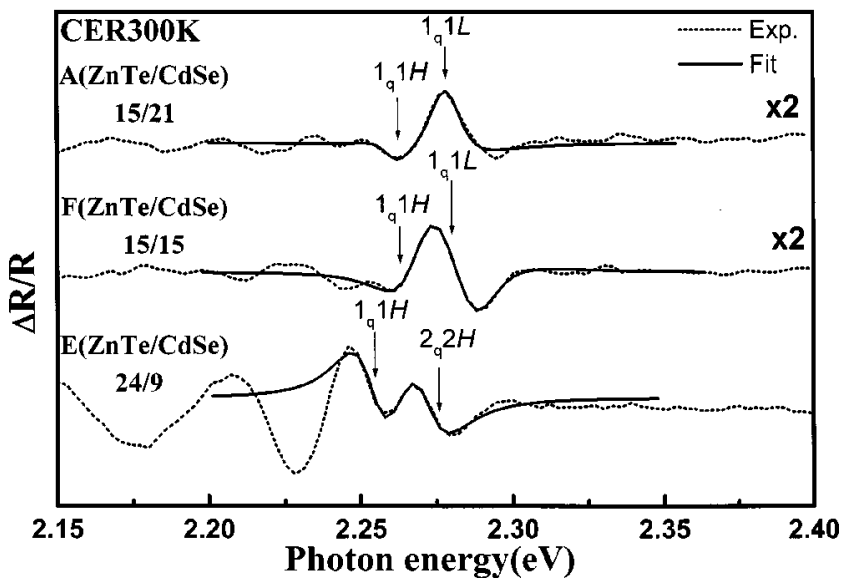

FIG. 4. The CER spectra of samples A, E, and F at $300 \mathrm{~K}$. The dotted lines represent experimental results, and solid lines are obtained by least-squares fits of a line-shape function.

the exciton absorption spectra, which associates with the transition from the first subband of heavy (light) holes in the valence-band barrier to the first subband of electrons in the conduction-band well (both in the CdSe layer). The oscillation features below and above the obvious excitonic lineshape signal in Figs. 2 and 3 are attributed to the interference effect or fringes of two light beams reflected from different interfaces of the sample. ${ }^{20}$ It is worth noting that the interference effect below the band gap of the $\mathrm{CdSe}(1.67 \mathrm{eV})$ can be observed up to $1 \mathrm{eV}$. We find that the interference effect distorts the line shape of the PR and CER features associated with multiple quantum wells, especially at the low-energy side. We cannot identify the higher-level transitions because the interference effect covers the higher-level transitions in the CdSe layer. Based on the reasons mentioned above, we only fit the line shape of the main transition features. In Fig. 2, we also clearly observe that the energy of the transition from quasibound to confined states in the CdSe layer increases as the CdSe width decreases (quantum confinement effect). In Fig. 3, we can see that the main transition position of samples D and E is nearly the same. It provides additional evidence showing that the observed transition is indeed in the CdSe layer because it does not change with the thickness of the ZnTe layer.

Figure 4 shows the CER spectra at room temperature for samples A, E, and F with the ZnTe layer width (CdSe layer width) of 15 (21), 24 (9), and $15 \mathrm{~nm}$ (15 nm), respectively. The features from confined to quasibound states are indicated by arrows. The notation of labeling transitions are the same as that of the PR spectra. The CER method was performed on all five samples that were previously measured by PR. The periodic features below the band gap of ZnTe (2.25 $\mathrm{eV}$ ) in Fig. 4 are due to the interference effect. Similarly, we just fit the apparent features above $2.25 \mathrm{eV}$.

Figure 5 shows the PC spectrum for sample B at room temperature. The PC spectrum contains two features labeled "a" and "b," at 1.7 and $2.25 \mathrm{eV}$, respectively. Based on the energy of these transitions, we attribute feature "b"to a type-I band-to-band transition between confined holes and quasiconfined electrons in the ZnTe layer as shown in Fig. 1. 


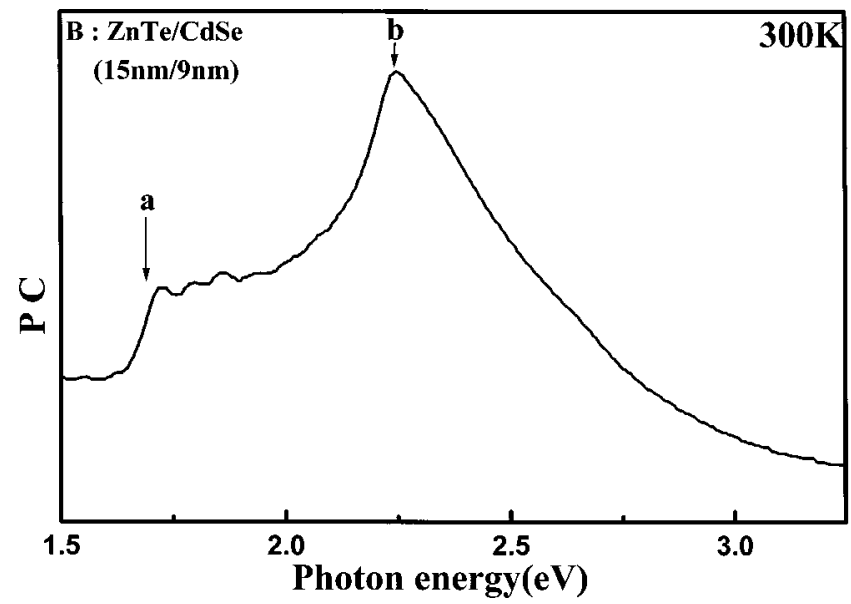

FIG. 5. The PC spectrum of sample B at $300 \mathrm{~K}$. Features "a" and " $b$ " discussed in the text are attributed to type-I transitions (either in the CdSe or ZnTe layer).

Similarly, feature "a" is interpreted as due to the type-I transition between quasiconfined holes and confined electrons in the CdSe layer as shown in Fig. 1. In addition, the weaker features around $1.75-2.0 \mathrm{eV}$ are due to the mixtures of the interference effect and higher-level transition, which can be observed more clearly at low temperature.

Figure 6 shows the PC spectrum for sample B at room temperature in the low-energy range. Starting from $0.9 \mathrm{eV}$, the PC spectrum shows a sharp increase and the signal is also influenced by the interference fringes. This behavior shows that there exists a strong transition around $1 \mathrm{eV}$, which corresponds to the type-II spatially indirect transition between electrons confined in the CdSe and holes confined in the $\mathrm{ZnTe}$ layers. The observation at room temperature indicates the strong localization of the carriers in our type-II superlattices.

Let us now compare the experimental results and theoretical calculations for the energies of the above-barrier transitions. The mechanism of localization of carriers at the abovebarrier region can be understood from basic quantummechanical theory. An electron wave traveling in the barrier

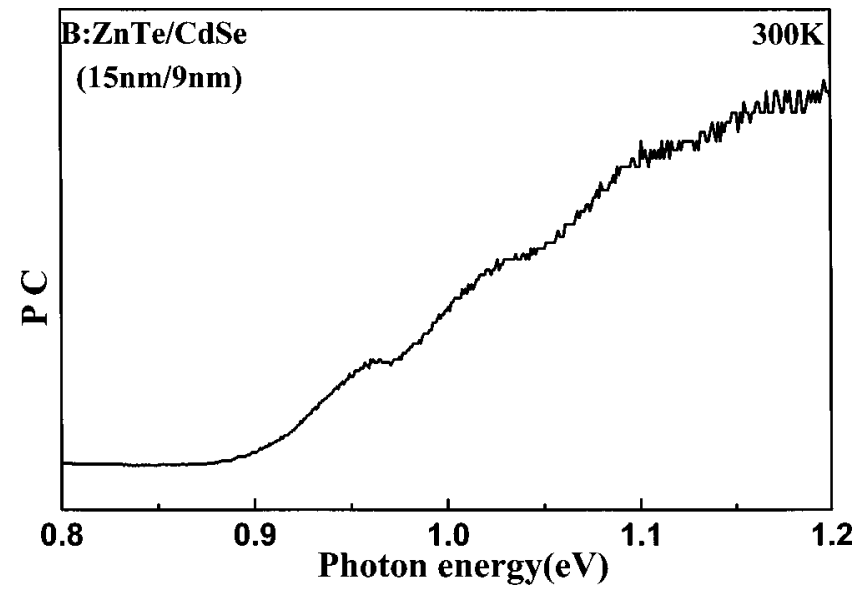

FIG. 6. The PC spectrum of sample $\mathrm{B}$ at $300 \mathrm{~K}$ in the lowenergy range.

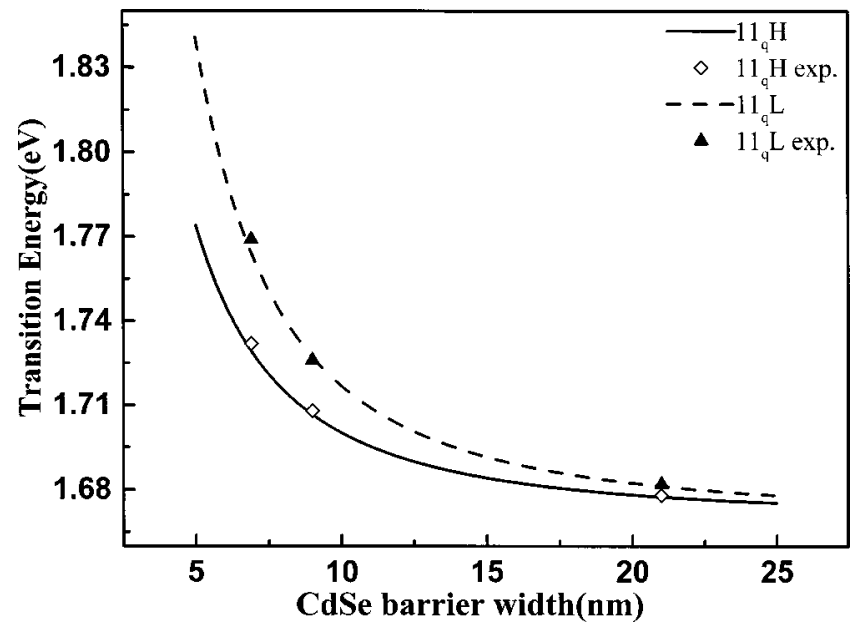

FIG. 7. A plot of the transition energies of the above-barrier states vs the CdSe barrier width of samples A, B, and C. The open squares and solid triangles are measured by photoreflectance. The solid and dashed lines represent the calculation using the constructive interference condition.

region experiences a reflection as it reaches the barrier-well interface, similarly to the case of below-barrier electrons traveling inside the well. Just as for electrons in the well, the constructive interference condition will have a strong effect on the shape of the above-barrier electron wave function. In the same way as a classical string with a different density, the standing wave will form as the constructive interference condition is satisfied. The formation of the standing wave implies the localization of the electron. The constructive interference condition is $k_{b} L_{b}=n \pi, 3,4,17,21,22$ where $k_{b}$ is the wave number in the barrier region, $L_{b}$ is the barrier width, and $n$ is an integer. The energy of the above-barrier state can be obtained by $E_{b}=\left(\hbar k_{b}\right)^{2} /\left(2 m_{b}^{*}\right)$, where $m_{b}^{*}$ is the effective mass of electrons or holes at the barrier region.

To complete the identification of features of the modulation spectra we performed a theoretical calculation based on the envelope function approximation. ${ }^{23}$ Numerical and experimental results of transition energies are summarized in Tables I and II. The parameters used in the calculations for the transition energies are as follows. The effective masses of electrons, heavy holes, and light holes in CdSe are $m_{e}(\mathrm{CdSe})=0.12, \quad m_{h h}(\mathrm{CdSe})=0.9, \quad$ and $\quad m_{l h}(\mathrm{CdSe})$ $=0.18 m_{0}$, respectively, where $m_{0}$ is the free-electron mass. ${ }^{24}$ For ZnTe, the electron, heavy-hole, and light-hole masses are $m_{e}(\mathrm{ZnTe})=0.124,{ }^{25} \quad m_{h h}(\mathrm{ZnTe})=0.64$, and $m_{l h}(\mathrm{ZnTe})=0.154 m_{0} \cdot{ }^{26}$ Exciton binding energies are included in the calculation for the transitions. ${ }^{24}$

Figure 7 shows the transition energies of the above-barrier states as a function of barrier width. The experimental results are represented by the open squares and solid triangles are for the photoreflectance. The conditions of localization of the wave function in the barrier region corresponding to the constructive interference condition $k_{b} L_{b}=n \pi$ are shown as solid and dashed curves in Fig. 7. We can clearly see that the solid and dashed curves can be used to describe well the transitions. The agreement between experimental and theoretical results supports our identification of the transition position. 


\section{CONCLUSIONS}

In summary, we report that the modulation spectroscopies and photoconductivity techniques can be used to observe the above-barrier quasibound-state transitions in type-II ZnTe/ CdSe superlattices at room temperature. We provide concrete evidence for the strong localization of the carrier waves in the barrier region. We show that the simple standing-wave condition proposed previously can give a clear physical picture on the above-barrier transition. Our results therefore also demonstrate that modulation and photoconductivity spectroscopies are very sensitive and powerful tools for the study of transitions in semiconductor quantum structures.

\section{ACKNOWLEDGMENTS}

We thank Dr. H. Luo and Dr. J. K. Furdyna for providing us the studied samples. This work was partially supported by the National Science Council of the Republic of China.
${ }^{1}$ J. E. Zucker, A. Pinczuk, D. S. Chemla, A. Gossard, and W. Wiegmann, Phys. Rev. B 29, 7065 (1984).

${ }^{2}$ J. J. Song, Y. S. Yoon, A. Fedotowsky, Y. B. Kim, J. N. Schulman, C. W. Tu, D. Huang, and H. Morkoc, Phys. Rev. B 34, 8958 (1986)

${ }^{3}$ F. C. Zhang, N. Dai, H. Luo, N. Samarth, M. Dobrowolska, J. K. Furdyna, and L. R. Ram-Mohan, Phys. Rev. Lett. 68, 3220 (1992).

${ }^{4}$ H. Luo, N. Dai, F. C. Zhang, N. Samarth, M. Dobrowolska, and J. K. Furdyna, Phys. Rev. Lett. 70, 1307 (1993).

${ }^{5}$ M. Nakayama, T. Fujita, and H. Nishimura, Superlattices Microstruct. 17, 31 (1995).

${ }^{6}$ S. H. Pan, H. Shen, Z. Huang, F. H. Pollak, W. Zhuang, Q. Xu, A. P. Roth, R. A. Masut, C. Lacelle, and D. Morris, Phys. Rev. B 38, 3375 (1988).

${ }^{7}$ C. Parks, A. K. Ramdas, M. R. Melloch, and L. R. Ram-Mohan, Phys. Rev. B 48, 5413 (1993).

${ }^{8}$ H. Luo, W. C. Chou, N. Samarth, A. Petrou, and J. K. Furdyna, Solid State Commun. 85, 691 (1993).

${ }^{9}$ J. J. Song, Y. S. Yoon, P. S. Jung, A. Fedotowsky, J. N. Schulman, C. W. Tu, J. M. Brown, D. Huang, and H. Morkoc, Appl. Phys. Lett. 50, 1269 (1987).

${ }^{10}$ H. Luo, N. Samarth, F. C. Zhang, A. Pareek, M. Dobrowolska, J. K. Furdyna, K. Mahalingam, N. Otsuka, W. C. Chou, A. Petrou, and S. B. Qadri, Appl. Phys. Lett. 58, 1783 (1991).

${ }^{11}$ N. Samarth, H. Luo, A. Pareek, F. C. Zhang, M. Dobrowolska, J. K. Furdyna, W. C. Chou, A. Petrou, K. Mahalingam, and N. Otsuka, J. Vac. Sci. Technol. B 10, 915 (1992).

${ }^{12}$ Y. S. Huang, H. Qiang, F. H. Pollak, G. D. Pettit, P. D. Kirchner,
J. M. Woodall, H. Stragier, and L. B. Sorensen, J. Appl. Phys. 70, 7537 (1991).

${ }^{13}$ F. H. Pollak and H. Shen, Mater. Sci. Eng., R. 10, 275 (1993).

${ }^{14}$ D. E. Aspnes, in Handbook on Semiconductors, edited by M. Balkanski (North-Holland, Amsterdam, 1980), Vol. 2, p. 109.

${ }^{15}$ F. H. Pollak, in Handbook on Semiconductors, edited by M. Balkanski (North-Holland, Amsterdam, 1994), Vol. 2, p. 527.

${ }^{16}$ O. J. Glembocki and B. V. Shanabrook, in Semiconductor and Semimetals, edited by D. G. Seiler and C. L. Littler (Academic, New York, 1992), Vol. 36, p. 222.

${ }^{17}$ F. C. Zhang, H. Luo, N. Dai, N. Samarth, M. Dobrowolska, and J. K. Furdyna, Phys. Rev. B 47, 3806 (1993).

${ }^{18}$ E. T. Yu, M. C. Phillips, J. O. McCaldin, and T. C. McGill, J. Vac. Sci. Technol. B 9, 2233 (1991).

${ }^{19}$ E. T. Lee, S. Stoltz, H. C. Chang, M. H. Na, H. Luo, and A. Petrou, Solid State Commun. 107, 177 (1998).

${ }^{20}$ D. Huang, D. Mui, and H. Morkoc, J. Appl. Phys. 66, 358 (1989).

${ }^{21}$ J. C. Fan, Y. F. Chen, D. Y. Lin, Y. S. Huang, M. C. Chen, and H. H. Lin, J. Appl. Phys. 86, 1460 (1999).

${ }^{22}$ J. C. Fan, Y. F. Chen, M. C. Chen, H. H. Lin, D. Y. Lin, and Y. S. Huang, Jpn. J. Appl. Phys., Part 1 36, 5448 (1997).

${ }^{23}$ G. Bastard and J. A. Brum, IEEE J. Quantum Electron. 22, 1625 (1986).

${ }^{24}$ Y. D. Kim, M. V. Klein, S. F. Ren, Y. C. Chang, H. Luo, N. Samarth, and J. K. Furdyna, Phys. Rev. B 49, 7262 (1994).

${ }^{25}$ D. Emanuelsson, M. Drechsler, D. M. Hofmann, Al. L. Efros, B. K. Mcyer, and B. Clerjaud, Solid State Commun. 90, 635 (1994).

${ }^{26}$ R. A. Stradling, Solid State Commun. 6, 665 (1968). 